ARTICLE

\title{
SLITRK5 is a negative regulator of hedgehog signaling in osteoblasts
}

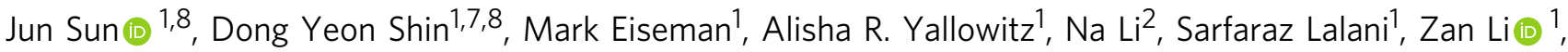 \\ Michelle Cung (10 ', Seoyeon Bok (10) ', Shawon Debnath', Sofia Jenia Marquez', Tommy E. White ${ }^{3}$, Abdul G. Khan ${ }^{3}$,

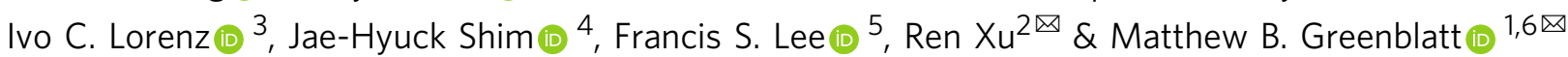

Hedgehog signaling is essential for bone formation, including functioning as a means for the growth plate to drive skeletal mineralization. However, the mechanisms regulating hedgehog signaling specifically in bone-forming osteoblasts are largely unknown. Here, we identified SLIT and NTRK-like protein-5(Slitrk5), a transmembrane protein with few identified functions, as a negative regulator of hedgehog signaling in osteoblasts. Slitrk5 is selectively expressed in osteoblasts and loss of Slitrk5 enhanced osteoblast differentiation in vitro and in vivo. Loss of SLITRK5 in vitro leads to increased hedgehog signaling and overexpression of SLITRK5 in osteoblasts inhibits the induction of targets downstream of hedgehog signaling. Mechanistically, SLITRK5 binds to hedgehog ligands via its extracellular domain and interacts with PTCH1 via its intracellular domain. SLITRK5 is present in the primary cilium, and loss of SLITRK5 enhances SMO ciliary enrichment upon SHH stimulation. Thus, SLITRK5 is a negative regulator of hedgehog signaling in osteoblasts that may be attractive as a therapeutic target to enhance bone formation.

\footnotetext{
${ }^{1}$ Department of Pathology and Laboratory Medicine, Weill Cornell Medicine, New York, NY, USA. ${ }^{2}$ State Key Laboratory of Cellular Stress Biology, School of Medicine, Xiamen University, Xiamen, Fujian, China. ${ }^{3}$ Tri-Institutional Therapeutics Discovery Institute, New York, NY, USA. ${ }^{4}$ Division of Rheumatology, Department of Medicine, University of Massachusetts Medical School, Worcester, MA, USA. ${ }^{5}$ Department of Psychiatry, Weill Cornell Medical College, New York, NY, USA. ${ }^{6}$ Research Division, Hospital for Special Surgery, New York, NY, USA. 'Present address: Research Center, LegoChem BioSciences, Inc., Daejeon, South Korea. ${ }^{8}$ These authors contributed equally: Jun Sun, Dong Yeon Shin. ${ }^{凶}$ email: xuren526@xmu.edu.cn; mag3003@med.cornell.edu
} 
C urrent treatments for osteoporosis all have major limitations, which includes rare but severe toxicities, limits on maximum duration of therapy, efficacy in certain anatomic sites, or inducing a low bone turnover state that is undesirable in some contexts, such as during repair of skeletal injury ${ }^{1}$. As many of these effects appear to be inherent to the molecular targets of these agents, ultimately addressing this issue will require identification of new therapeutic targets to increase bone formation. In this respect, it is notable that while the Hedgehog (Hh) pathway plays a fundamental role in bone development and homeostasis, it has yet to be therapeutically harnessed for skeletal disorders. The Hh pathway is initiated through the binding of $\mathrm{Hh}$ ligands to the transmembrane receptor Patched1 (PTCH1), relieving its repressive effects on Smoothened (SMO). Activated SMO moves to the primary cilium where it acts through several intermediates to ultimately activate GLI family member transcription factors ${ }^{2}$. GLI transcription factors, primarily GLI1 and GLI2, subsequently enter the nucleus to activate downstream target gene expression, which includes transcriptional feedback regulation of Hh pathway components themselves (Ptch1, Gli1, and Hhip $)^{3}$. Disruption of $\mathrm{Hh}$ signaling leads to multiple bone defects. Ihh deficient mice display dwarfism with disruptions in growth plate structure ${ }^{4}$. Ptch1 haploinsufficiency leads to increased bone mass in mice, which is driven by enhanced osteoblast responsiveness to Runx $2^{5}$. Conversely, Gli1 haploinsufficiency causes decreased bone mass and impaired fracture healing in mice ${ }^{2,6}$. In addition, mice with an upregulation of $\mathrm{Hh}$ signaling in mature osteoblasts display both increased bone formation and excess RANKL-driven osteoclastogenesis, which results in enhanced bone resorption and reduced bone mass ${ }^{7}$. Given the central role of the Hh pathway in osteoblasts, proper regulation of this pathway is essential. However, little is known about how the responses of osteoblasts to Hh ligands is "finetuned".

We performed an initial screen for transmembrane proteins displaying selective expression in osteoblasts, as this gene set will be enriched for novel receptors or co-receptors that may be druggable, and identified SLITRK5 as a co-receptor that regulates Hh signaling. The SLITRK family, composed of SLITRK1 through SLITRK6, are type I single pass transmembrane proteins. Their nomenclature is based on containing N-terminal extracellular leucine-rich repeat (LRR) domains, similar to those of Slit proteins, and an intracellular carboxyl terminus that has sequence similarity to neurotrophin receptors $(\text { Trks })^{8}$. While there have been relatively few studies of SLITRK family members, studies to date show that Slitrks are highly expressed in the central nervous system and play roles in neuronal survival, neurite growth and synapse formation ${ }^{9-11}$. In mice, genetic deletion of Slitrk5 leads to defects in corticostriatal neurotransmission and obsessive-compulsive disorder-like behaviors ${ }^{12}$. Here we find that loss of Slitrk5 enhances osteoblast differentiation and function in vitro and in vivo by directly regulating Hh signaling in osteoblasts. Slitrk 5 represses the expression of downstream Hh target genes by its direct interactions with Shh and Ptch1. Taken together, this identifies Slitrk5 as a novel Hh co-receptor that represses Hh signaling specifically in osteoblasts to regulate bone formation.

\section{Results}

Slitrk5-deficiency promotes osteoblastogenesis in vitro. To identify druggable targets that may increase bone formation, we screened gene expression data for transmembrane proteins showing selective expression in osteoblasts ${ }^{13,14}$. Slitrk5 was identified as specifically expressed in osteoblasts, apart from its robust expression in the nervous system, and it has only very modest expression in osteoclasts and bone marrow cells (Fig. 1a and Supplementary Fig. 1a). To confirm that SLITRK5 shows selective expression in osteoblasts, a reporter mouse with an insertion of a beta-galactosidase cassette into a Slitrk5 intronic sequence was used $^{12}$. Beta-galactosidase staining confirmed Slitrk5 expression in osteoblasts residing on the trabecular bone surface and periosteum, showing colocalization of staining with the osteoblast marker osteopontin (OPN) (Fig. 1b and Supplementary Fig. 1b). To investigate the role of Slitrk5 in osteoblast differentiation, calvarial osteoblasts isolated from WT and Slitrk5 ${ }^{-/}$mice were cultured under osteoblast differentiation conditions. Slitrk5 $5^{-/-}$ osteoblasts displayed enhanced differentiation and increased mineralization capacity as indicated by alizarin red staining (Fig. 1c). Likewise, an increase in alkaline phosphatase (ALP) activity was observed in Slitrk5 ${ }^{-/}$- osteoblasts (Fig. 1d). In addition, we measured the mRNA levels of osteoblast marker genes after 6 and 12 days of differentiation. Consistent with an increase in ALP and mineralization activity, expression of characteristic osteoblast transcripts, including Runx2,Sp7,Bsp, Ocn, and $A l p l$, were all markedly increased in Slitrk5 $5^{-/-}$osteoblasts (Fig. 1e). Thus, Slitrk5 is selectively expressed in osteoblasts and Slitrk5 represses osteoblast differentiation in vitro.

Slitrk5 is a negative regulator of hedgehog signaling in osteoblasts. A previous study showed that Slitrk5 is critical for brain-derived neurotrophic factor (BDNF)-dependent signaling in neural cells ${ }^{15}$. To assess whether Slitrk5 regulates osteoblast differentiation through BDNF signaling, WT and Slitrk5 ${ }^{-/-}$ calvarial osteoblasts were treated with BDNF over the course of osteoblast differentiation. BDNF did not impact osteoblast differentiation in either WT or Slitrk5 $5^{-/-}$cells (Fig. 2a). In addition, WT or Slitrk5 $5^{-1-}$ cells were treated with K252A, an inhibitor of the TRK family of receptor tyrosine kinases, that blocks signaling by BDNF and other TRK family ligands. TRK inhibition was associated with decreased, not increased, ALP levels (Supplementary Fig. 2a). Thus, Slitrk5 likely controls osteoblast differentiation through an alternative mechanism.

A prior study reported that Slitrk5 expression was significantly increased in a Hh-induced mouse model of medulloblastoma ${ }^{16}$ (Supplementary Fig. 2b). Given that many $\mathrm{Hh}$ signaling components or regulators are themselves targets of Hh signaling, this raised the possibility that SLITRK5 regulates Hh activity. To explore if $\mathrm{Hh}$ activity is enhanced in a manner consistent with the augmented osteoblast differentiation in Slitrk5 $5^{-1-}$ cells, the expression of $\mathrm{Hh}$ target genes were examined in cultured osteoblasts. As shown in Fig. 2b, the level of these genes was upregulated in Slitrk5 $5^{-1-}$ osteoblasts, suggesting that $\mathrm{Hh}$ signaling is augmented in the absence of SLITRK5. Knockdown of SLITRK5 in Saos2 human osteoblast-like cells similarly increased the expression level of marker genes of Hh pathway activity, including Gli1, Gli2 and Ptch1 (Supplementary Fig. 2c). Conversely, over-expression of SLITRK5 had the opposite effect and suppressed responses to $\mathrm{SHH}$, shown by decreased levels of the Hh reporter genes Gli1, Ptch1, and Hhip (Fig. 2c). Similarly, the activation of a Gli1-responsive reporter gene was suppressed by overexpression of Slitrk5 (Fig. 2d). These data identify Slitrk5 as a negative regulator of Hedgehog signaling in osteoblasts.

Activation of hedgehog signaling can induce osteoblast differentiation ${ }^{17}$. To investigate whether Slitrk5-deficient osteoblasts are more sensitive to SHH stimulation, WT and Slitrk5 ${ }^{-1-}$ osteoblasts were treated with different doses of $\mathrm{SHH}$ and osteoblast differentiation was assessed by ALP activity. Loss of Slitrk5 amplified the effect of SHH on osteoblast differentiation, effectively producing an approximately 8-fold increase in $\mathrm{Hh}$ ligand potency (Fig. 2e). Similarly, knockdown of Slitrk5 in calvarial osteoblasts also increased SHH-induced ALP activity in 
a

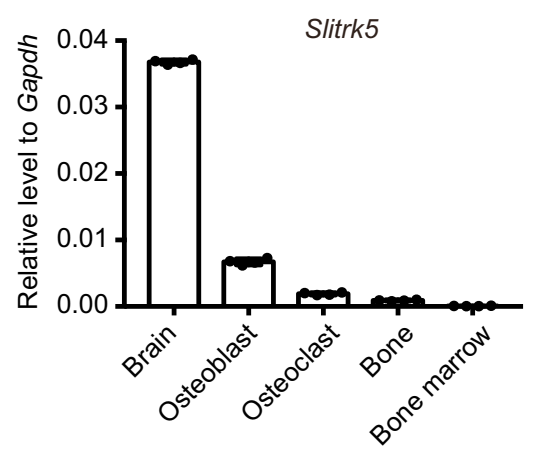

C

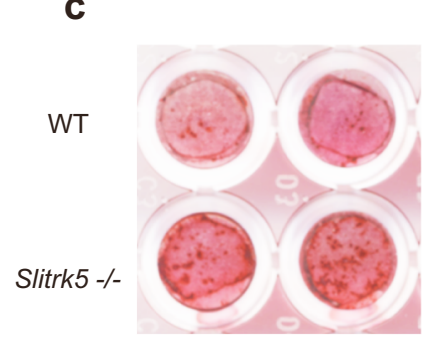

d

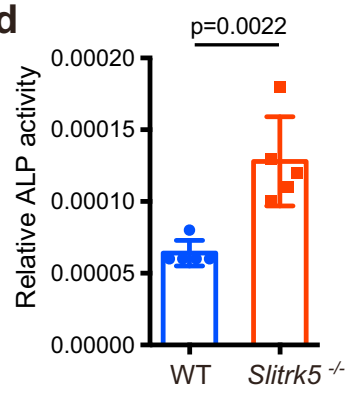

b

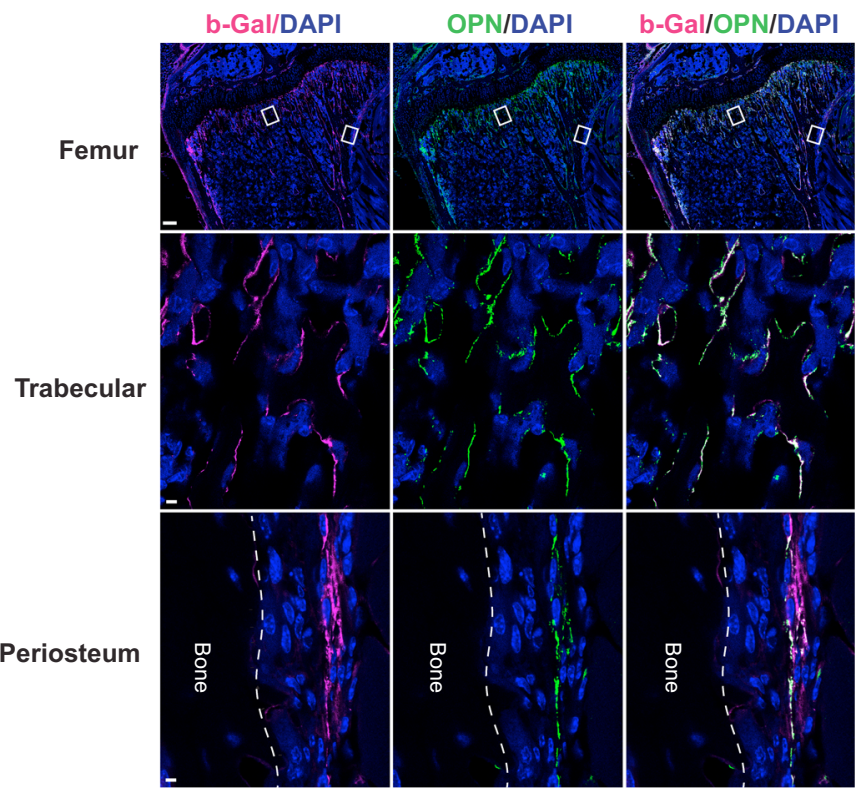

e

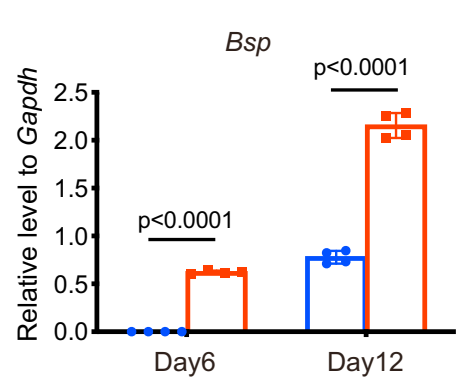

Sp7

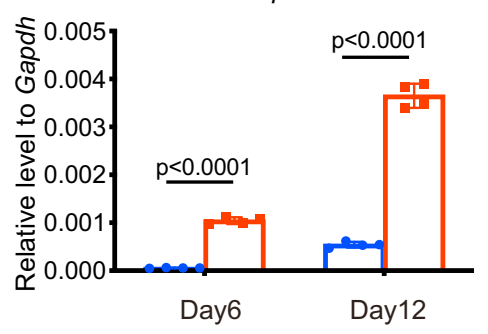

Ocn

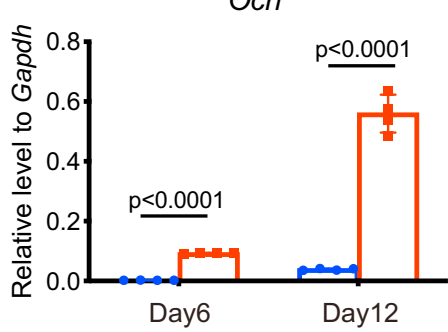

Runx2

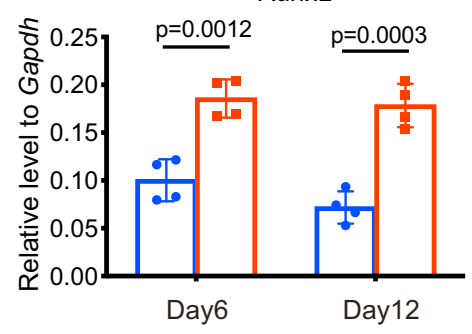

$\begin{array}{ll}\text { Alpl } & \square \text { WT } \\ & \square \text { Slitrk5 } \%\end{array}$

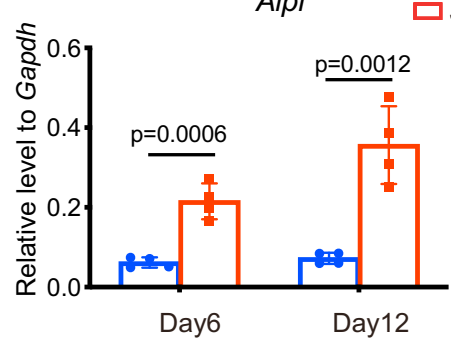

Slitrk5

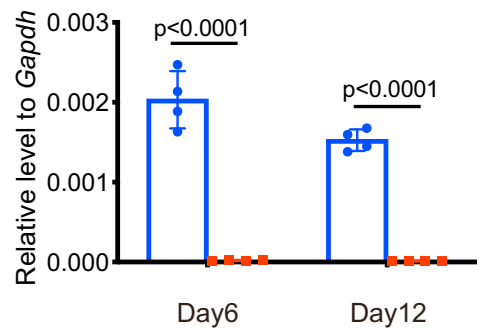

Fig. 1 Slitrk 5 is expressed in osteoblasts and negatively regulates osteoblastogenesis. a Expression of Slitrk5 mRNA in indicated cells or tissues. Data are presented as mean \pm s.d. $n=4$ biologically independent samples. $\mathbf{b}$ Immunofluorescence staining of mouse femur sections with anti-beta-galactosidase and OPN antibodies, demonstrating the expression of Slitrk5 in osteoblasts. Data are representative of two independent experiments, scale bar $=200 / 10 /$ $10 \mu \mathrm{m}$. c, d Primary osteoblasts from WT and Slitrk5 ${ }^{-/}$- mice were cultured in osteoblast differentiation medium. Mineralization activity was assessed by alizarin red staining (c) at day 14 of differentiation. Alkaline phosphatase (ALP) activity was measured at day 8 of differentiation (d). Data in (d) are presented as mean \pm s.d. $n=5$ biologically independent samples, two-tailed unpaired $t$ test. e RT-PCR analysis of Slitrk5 and osteoblast marker genes expression at day 6 and day 12 of differentiation in WT and Slitrk5-/- osteoblasts during differentiation. $n=4$ biologically independent samples, two-tailed unpaired $t$ test. Data are presented as mean \pm s.d.

a manner that was responsive to the degree of knockdown (Fig. 2f, g). Thus, loss of Slitrk5 leads to increased hedgehog responsiveness during osteoblast differentiation.

SLITRK5 interacts with SHH and PTCH1. Activation of the hedgehog signaling pathway involves Hh ligand binding to
PTCH1 and activation of SMO at the cell membrane which is followed by the activation of GLI inside the cell ${ }^{3}$. To delineate which components of the Hh signaling pathway are regulated by Slitrk5, we first treated WT and Slitrk5 $5^{-1-}$ calvarial osteoblasts with purmorphamine, a direct SMO agonist, and osteoblast differentiation was assessed by ALP activity. While purmorphamine 
a
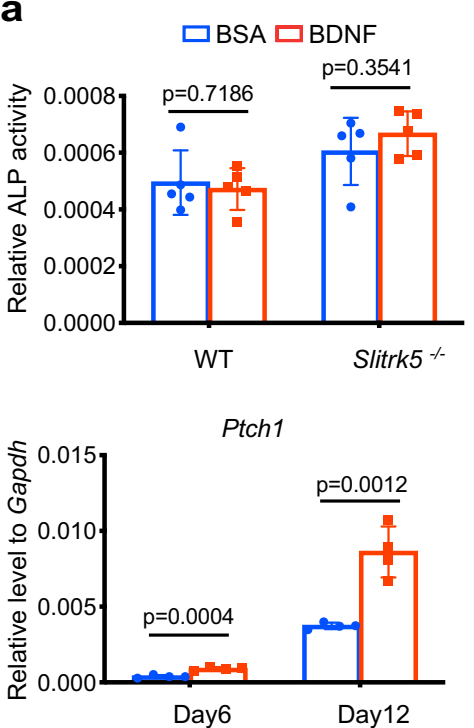

C
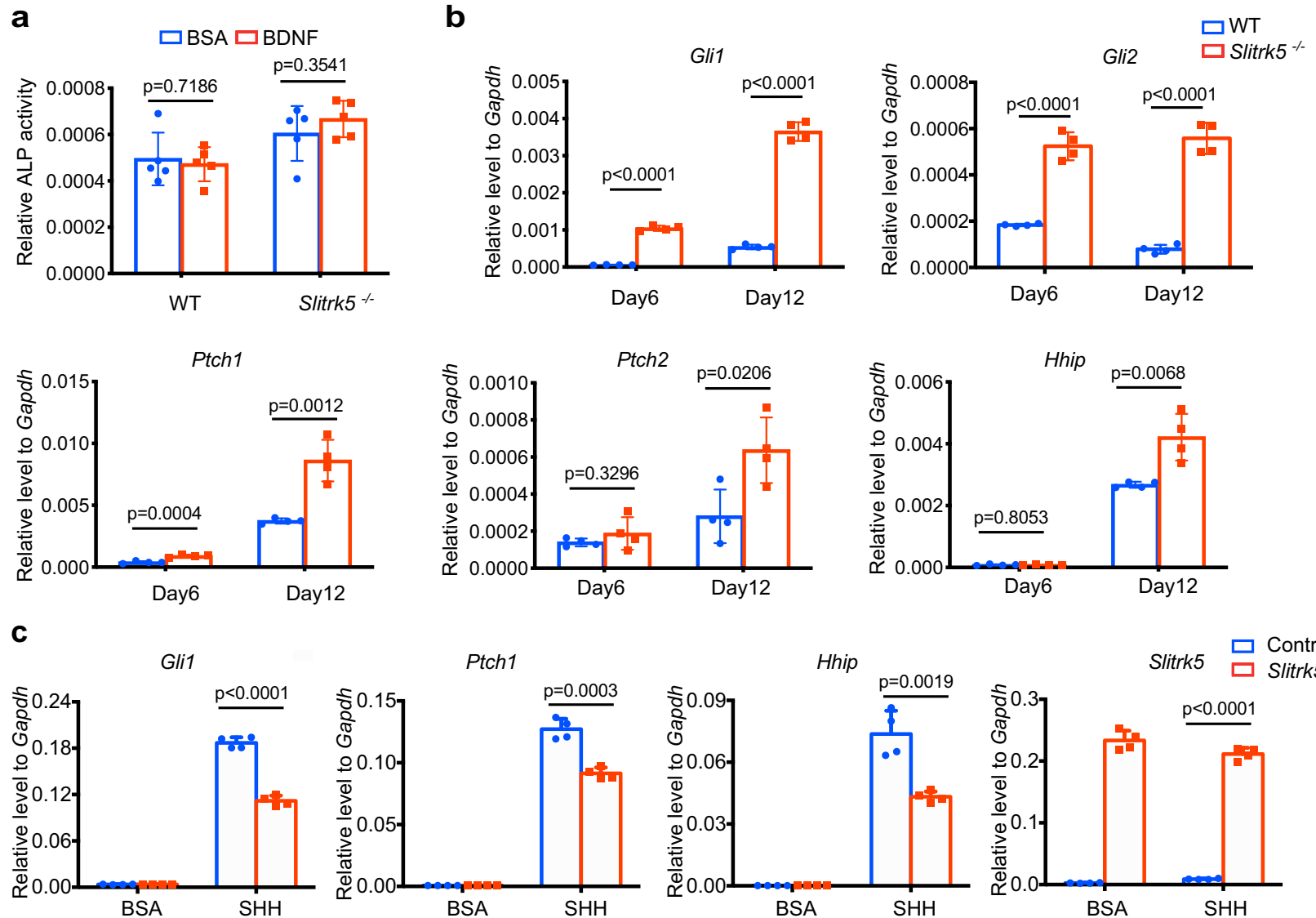

d
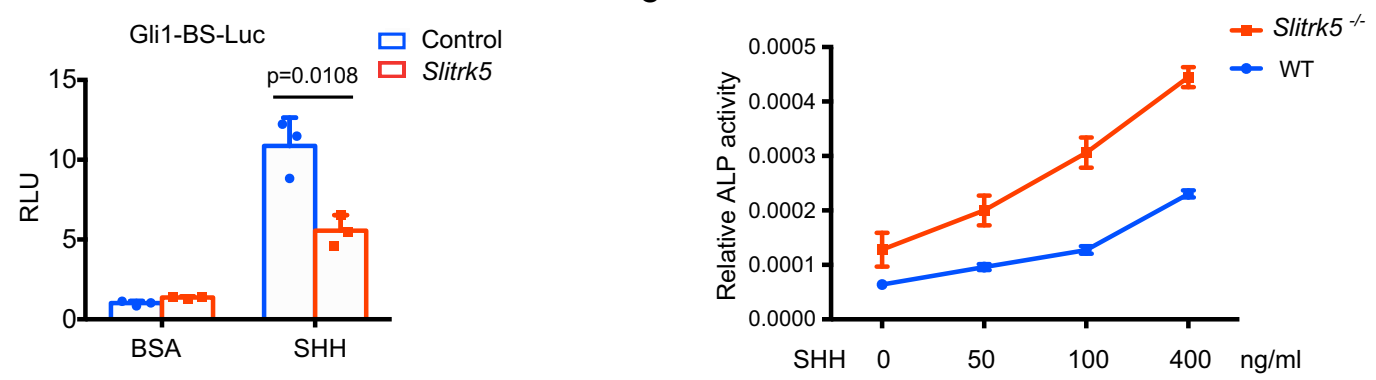

$\mathbf{f}$

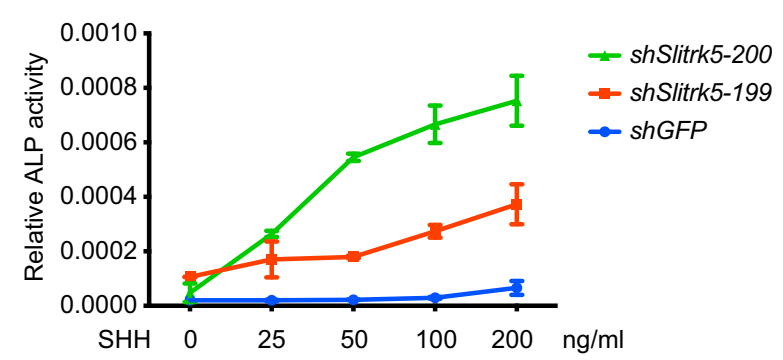

promoted osteoblast differentiation as expected, there was no difference in response between WT and Slitrk5 ${ }^{-1-}$ cells, indicating that SLITRK5 functions upstream of SMO, likely at the level of the PTCH1 complex (Fig. 3a and Supplementary Fig. 3a). As SLITRK5 is a transmembrane protein, we next investigated whether SLITRK5 binds to $\mathrm{SHH}$, demonstrating an interaction between Flag-SLITRK5 and $\mathrm{SHH}$ in immunoprecipitation

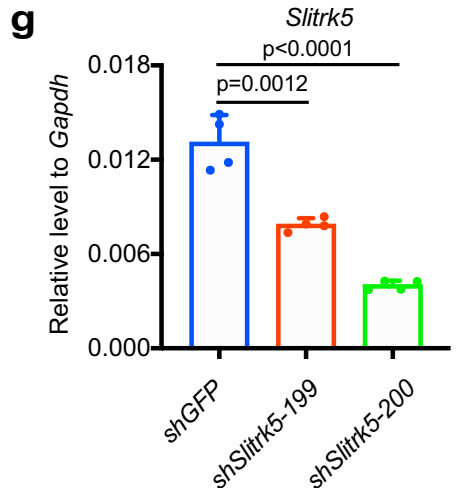

assays (Fig. 3b). Among members of the SLITRK family, this ability to interact with $\mathrm{SHH}$ is restricted to SLITRK5, as SLITRK1 and SLITRK6 displayed no evidence of SHH interaction (Supplementary Fig. 3b, c). In keeping with this, SLITRK1 and SLITRK6 lacked the ability of SLITRK5 to suppress SHH responses in an enforced expression system (Supplementary Fig. 3d). 
Fig. 2 Slitrk5 is a negative regulator of hedgehog signaling in osteoblasts. a Primary osteoblasts from WT and Slitrk5-/- mice were treated with BSA control or $40 \mathrm{ng} / \mathrm{ml} \mathrm{BDNF}$ and cultured in osteoblast differentiation medium. ALP activity was measured at day 6 of osteoblast differentiation. Data are presented as mean \pm s.d. $n=5$ biologically independent samples, two-tailed unpaired $t$ test. $\mathbf{b}$ RT-PCR analysis of hedgehog signaling related gene expression at day 6 and day 12 of differentiation in WT and Slitrk5 $5^{-/-}$osteoblasts. $n=4$ biologically independent samples, two-tailed unpaired $t$ test. c $\mathrm{C} 3 \mathrm{H} 10 \mathrm{~T} 1 / 2$ cells transfected with either control or Slitrk5 overexpression vectors were treated with BSA or $100 \mathrm{ng} / \mathrm{ml} \mathrm{SHH}$ in serum-free medium for 48 h. Gli1, Ptch1, Hhip, and Slitrk5 mRNA levels were measured by RT-PCR. $n=4$ biologically independent samples, two-tailed unpaired $t$ test. $\mathbf{d}$ C3H10T1/2 cells transfected with GLI1-luc/Renilla together with either control vector or Slitrk5 overexpression vector were treated with BSA or $100 \mathrm{ng} / \mathrm{ml}$ SHH in serum-free medium for $36 \mathrm{~h}$. Luciferase activity was measured to assess Hh signaling. $n=3$ biologically independent samples, two-tailed unpaired $t$ test. e Primary osteoblasts from WT and Slitrk5 $5^{-1}$ mice were treated with different doses of SHH and cultured in osteoblast differentiation medium. ALP activity was measured at day 8 of differentiation. Data are presented as mean \pm s.d. $n=5$ biologically independent samples. $\mathbf{f}$ Primary osteoblasts transduced with Slitrk5 or Gfp shRNAs were treated with the indicated doses of SHH and cultured in osteoblast differentiation medium. ALP activity was measured at day 8 of osteoblast differentiation. Data are presented as mean \pm s.d. $n=2$ biologically independent samples. $\mathbf{g}$ Knockdown efficiency of Slitrk5 was validated by qRT-PCR. $n=4$ biologically independent samples, two-tailed unpaired $t$ test.

SLITRK5 is a single-pass transmembrane protein with an extracellular domain containing two LRR domains and intracellular carboxyl terminus. To further map the interaction region within SLITRK5, we generated a series of SLITRK5 truncation mutants, finding that a SLITRK5 construct lacking the entire extracellular domain, but not a construct lacking the first extracellular LRR domain alone, lost the ability to bind to $\mathrm{SHH}$ (Fig. 3c). Thus, SHH binds to either the second extracellular LRR domain or intervening linker sequences.

To address whether the interaction between SLITRK5 and $\mathrm{SHH}$ is direct, a cell-free binding assay was performed where a recombinant human SLITRK5 extracellular domain fragment was affixed to a solid phase and interaction with an epitope-tagged His-SHH was assayed via anti-His HRP (Fig. 3d). This approach revealed a direct interaction between $\mathrm{SHH}$ and the SLITRK5 extracellular domain. This was also confirmed by surface plasmon resonance which showed binding of a SLITRK5 extracellular domain fragment to $\mathrm{SHH}$ with a $\mathrm{Kd}$ of $\sim 40 \mathrm{nM}$ (Fig. 3e).

As these findings suggest that SLITRK5 may function as a $\mathrm{SHH}$ co-receptor, we examined if SLITRK5 may interact with the primary $\mathrm{SHH}$ receptor, PTCH1. Indeed, overexpressed HAPTCH1 and Flag-SLITRK5 displayed a bidirectional, reciprocal interaction in immunoprecipitation assays in both HEK293 and C3H10t1/2 cells (Fig. 3f, g and Supplementary Fig. 3e). This interaction between SLITRK5 and PTCH1 was not affected by overexpression of SHH (Fig. 3f, g and Supplementary Fig. 3e). Using multiple SLITRK5 truncation mutants, the interaction between SLITRK5 and PTCH1 was mapped to the intracellular domain of SLITRK5 (Fig. 3h). Thus, SLITRK5 binds to SHH through its extracellular domain and to PTCH1 through its intercellular domain.

SLITRK5 is located at the primary cilium and regulates SMO ciliary enrichment upon SHH stimulation. Hedgehog signaling is functionally linked to the primary cilia in vertebrates. To investigate whether SLITRK5 is located at the cilium, we transduced primary osteoblasts with constructs encoding Flag-Slitrk5 and performed anti-Flag immunofluorescence. In line with a previous report ${ }^{15}$, SLITRK5 showed a punctate distribution in the cytoplasm (Fig. 4a). Moreover, SLITRK5 additionally localized to the primary cilium, as indicated by the colocalization of the primary ciliary marker acetylated tubulin and SLITRK5 (Fig. 4a). SHH stimulation did not significantly increase the amount of SLITRK5 in the primary cilium (Fig. 4b). As ciliary localization of SMO and PTCH1 is linked to the activation of hedgehog signaling, we next examined whether SLITRK5 affects ciliary localization of SMO and PTCH1. Primary Slitrk5 $5^{-1-}$ and WT osteoblasts were infected with Smo-GFP or Ptch1-Flag virus and the ciliary traffic of SMO and PTCH1 was followed in response to SHH stimulation. The ciliary localization of SMO and PTCH1 was comparable in Slitrk5 $5^{-1-}$ and WT cells in the absence of SHH stimulation (Fig. 4c-f). Both Slitrk5 ${ }^{-1-}$ and WT osteoblasts also showed a similar decrease in the PTCH1 ciliary localization after SHH simulation. In contrast, SHH-induced SMO ciliary enrichment was enhanced in Slitrk5 $5^{-1-}$ cells (Fig. 4c-f). This enhancement in SHH-induced SMO ciliary recruitment is consistent with the overall enhanced SHH signaling seen the absence of SLITRK5 and with the results of purmorphamine stimulation indicating that SLITRK5 acts upstream of SMO, likely regulating signaling between PTCH1 and SMO.

Slitrk5-deficiency promotes postnatal bone formation and fracture healing in mice. Hedgehog signaling in osteoblasts plays an important role in the postnatal accrual of bone mass ${ }^{18}$. To examine whether regulation of hedgehog signaling by SLITRK5 observed in vivo is relevant to the regulation of bone mass in vivo, the expression of hedgehog signaling target genes was examined, finding increases consistent with enhanced hedgehog signaling activity in the tibias of Slitrk5 $5^{-1-}$ mice (Fig. 5a, b). To explore the role of Slitrk5 in bone formation, dynamic histomorphometry analysis was performed on vertebrae from 7 -week-old WT and Slitrk $5^{-1-}$ mice. The mineral apposition rate and bone formation rate in trabecular and cortical bone were both increased in Slitrk5 $5^{-1-}$ mice (Fig. $5 \mathrm{c}-\mathrm{f}$ ). Consistent with this, the number of osteoblasts on the bone surface was also increased in Slitrk5 $5^{-1-}$ mice (Fig. $5 \mathrm{~g}, \mathrm{~h}$ ). In addition, osteoclast numbers were increased in Slitrk $5^{-1-}$ mice (Supplementary Fig. 4a, b). In line with this concurrent increase in both bone formation and osteoclastogenesis, overall bone mass was not changed in Slitrk5 $5^{-1-}$ mice as assessed by microCT (Supplementary Fig. 4c, d), a finding also consistent with reports that enhanced hedgehog signaling in osteoblasts results in excessive bone resorption ${ }^{7}$. Thus, loss of Slitrk5 in mice results in a high bone turnover state with increased bone formation and bone resorption.

Hedgehog also plays a critical role during fracture healing. $\mathrm{SHH}$ is expressed at fracture sites and regulates osteoblast proliferation and differentiation ${ }^{19}$. Activation of $\mathrm{Hh}$ signaling was reported to enhance fracture healing, raising the possibility that augmenting $\mathrm{Hh}$ signaling will improve skeletal repair ${ }^{20}$. To examine the role of Slitrk5 in fracture healing, we performed femoral fractures in WT and Slitrk5 $5^{-1-}$ mice. The callus was harvested and analyzed by $\mu \mathrm{CT} 3$ weeks post-fracture, finding that callus mineralization was increased in Slitrk5 $5^{-1-}$ mice (Fig. 5i, j). Accordingly, histology also showed increased bone in the callus of Slitrk5 $5^{-1-}$ mice (Fig. 5k). Consistent with the increased callus bone formation observed, Colla1 mRNA level was increased in the fracture callus of Slitrk $5^{-1-}$ mice, indicating enhanced osteoblast differentiation (Fig. 5l, m). Furthermore, the increased hedgehog signaling was observed in callus region of 
a

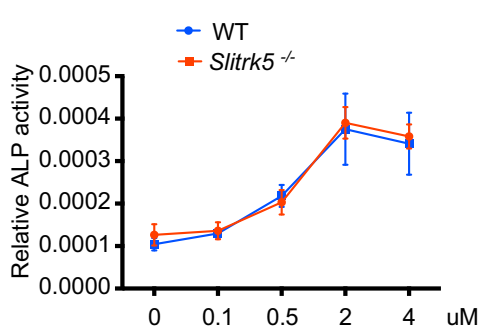

C

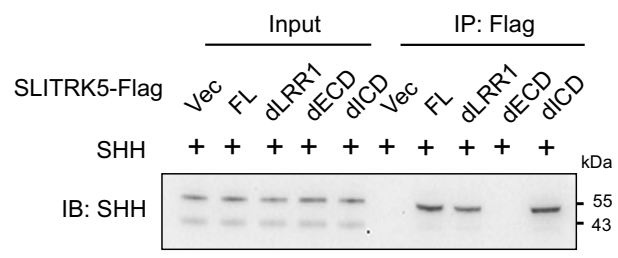

IB: Flag
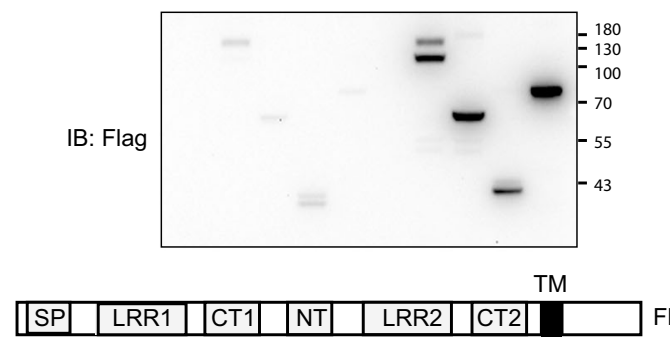

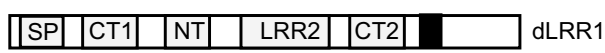

\begin{tabular}{|l|l|l}
\hline $\mathrm{SP}$ & \\
$\mathrm{dECCD}$
\end{tabular}

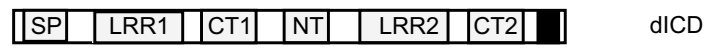

f

PTCH1-HA -+-+++

SLITRK5-Flag --+-+-+

$\mathrm{SHH}-\mathrm{C}^{-}+-++$

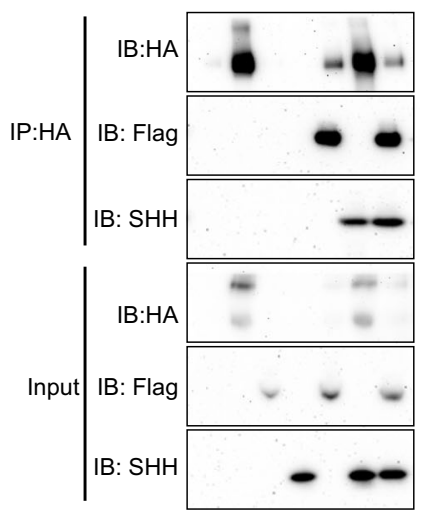

g

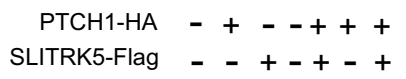

SLITRK5-Flag - - + + -+

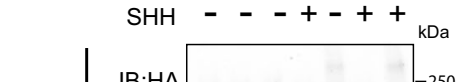

b

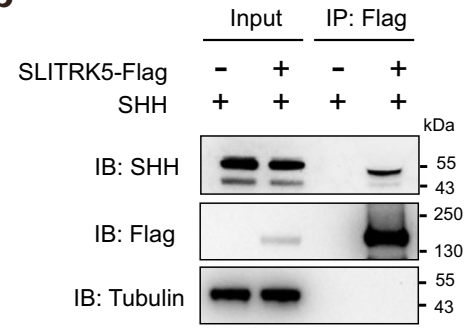

d

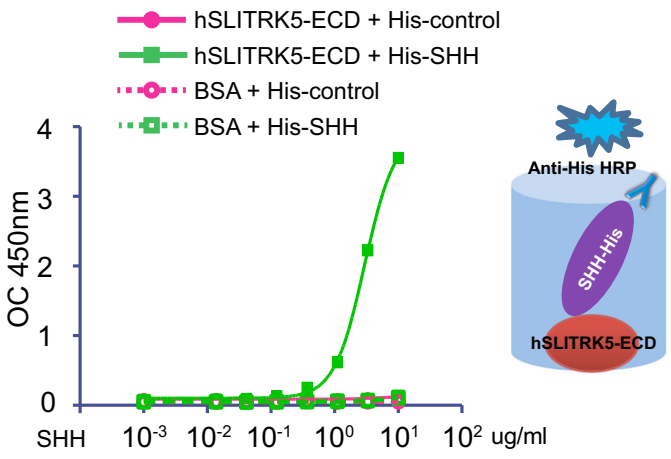

e

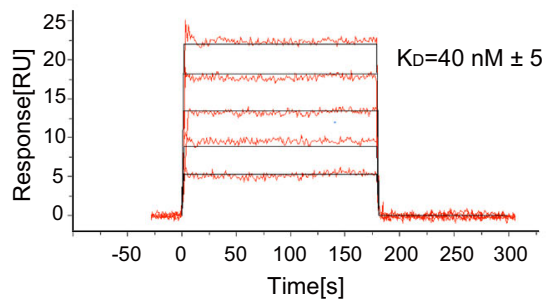

h

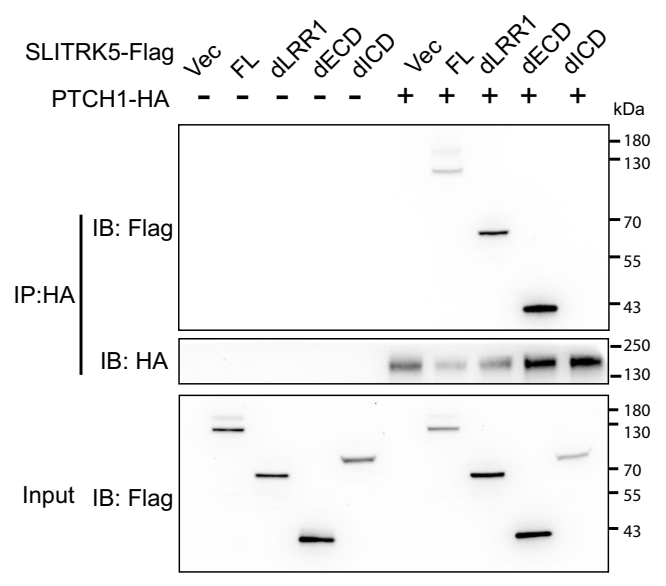

Fig. 3 SLITRK5 interacts with SHH and PTCH1. a Primary osteoblasts from WT and Slitrk5 ${ }^{-/}$- mice were treated with the indicated doses of Purmorphamine and cultured in osteoblast differentiation medium. ALP activity was measured at day 3 of differentiation. Data are presented as mean \pm s.d. $n=6$ biologically independent samples. b Co-immunoprecipitation of Flag-SLITRK5 and SHH in HEK293T cells. Data are representative of four independent experiments. c Co-immunoprecipitation of SHH and Flag-tagged SLITRK5 truncation mutants in HEK293T cells. Data are representative of three independent experiments. d ELISA assay showing the interaction of hSLITRK5-ECD and SHH. e Surface Plasmon Resonance analysis of the binding of SHH to SLITRK5. SHH was injected over a SLITRK5 surface at 125, 62.5, 31.25, 15.625, and 7.8125 nM. f, g Co-immunoprecipitation of Flag-SLITRK5 and HAPTCH1 in HEK293T cells with BSA or SHH treatment. Data are representative of two independent experiments. $\mathbf{h}$ Co-immunoprecipitation of PTCH1 and Flag-tagged SLITRK5 truncation mutants in HEK293T cells. Data are representative of three independent experiments. 
a

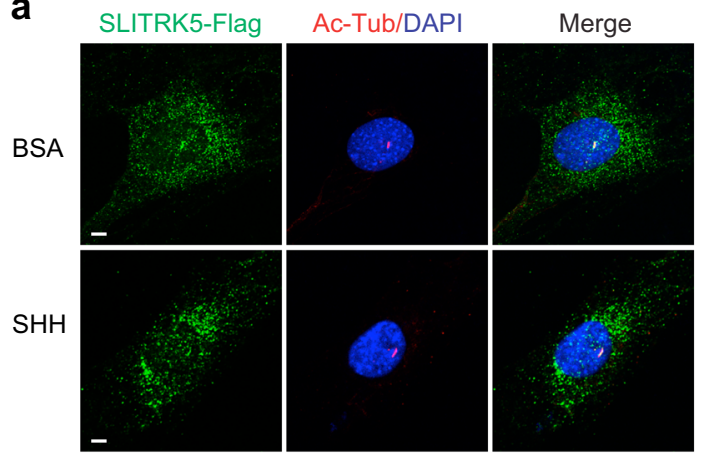

b

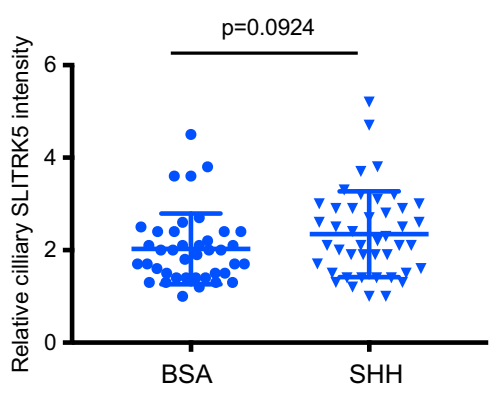

C

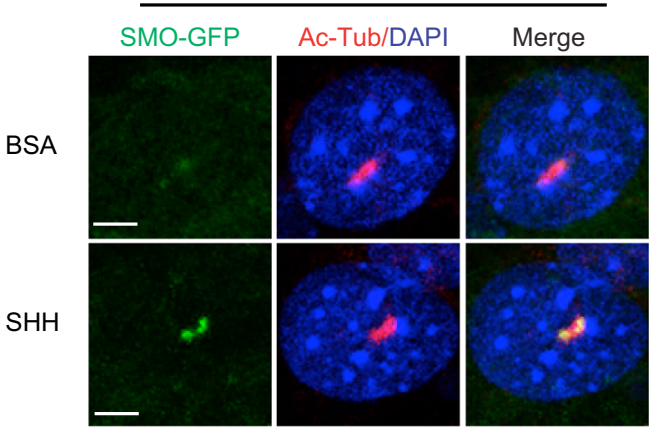

Slitrk5 $\%$

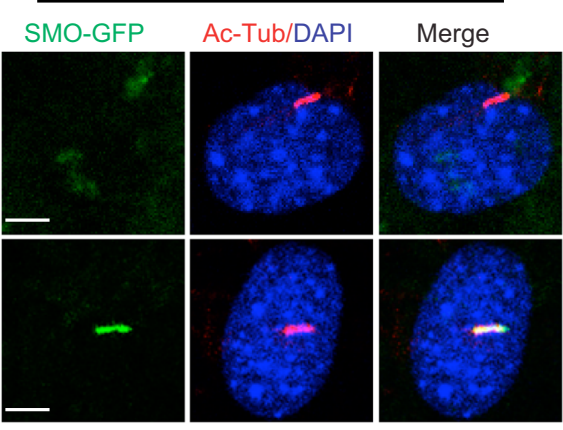

d WT

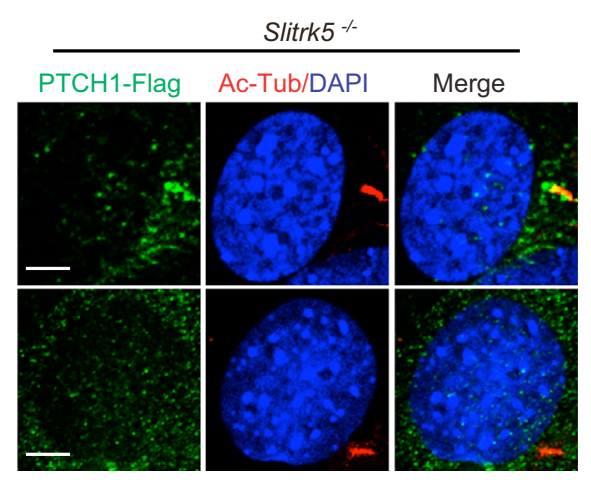

e

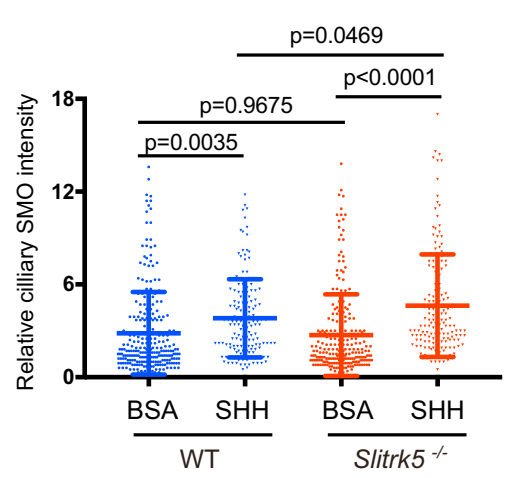

f

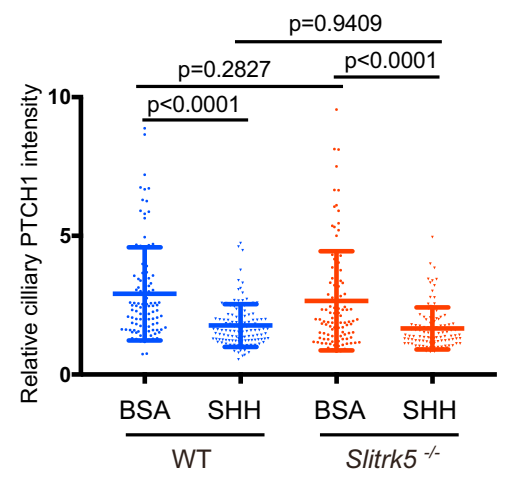

Fig. 4 SLITRK5 regulates SMO ciliary enrichment upon SHH stimulation. a Immunostaining for SLITRK5 (anti-Flag antibody, Green) and acetylated tubulin (Red) in osteoblasts treated with BSA or SHH for $4 \mathrm{~h}$. Data are representative of three independent experiments, scale bar $=5 \mu \mathrm{m}$. $\mathbf{b}$ Quantification of the relative fluorescence intensity of ciliary SLITRK5-flag. $n=40-50$ cells per group. Data are presented as mean \pm s.d, two-tailed unpaired $t$ test. c, $\mathbf{d}$ Ciliary localization of SMO-GFP (c) and PTCH1- Flag (d) in WT and Slitrk5-/- osteoblasts treated with BSA or SHH for $4 \mathrm{~h}$. Data are representative of three independent experiments, Scale bar $=5 \mu \mathrm{m}$. e, $\mathbf{f}$ Quantification of the relative fluorescence intensity of ciliary SMO-GFP (e) and PTCH1-Flag (f) in WT and Slitrk5 ${ }^{-/-}$osteoblasts treated with BSA or SHH for 4 h. $n=100-300$ cells per group. Data are presented as mean \pm s.d, One-way ANOVA ( $P<$ 0.0001) followed by a Tukey's post hoc test. 
a

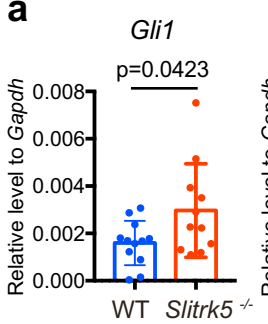

C

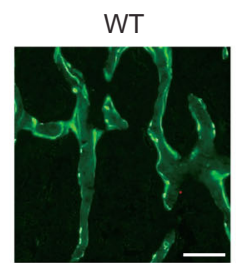

d

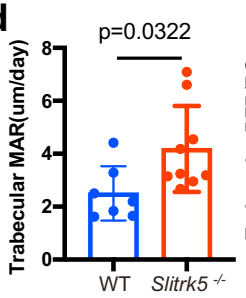

i

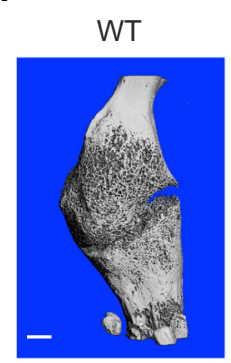

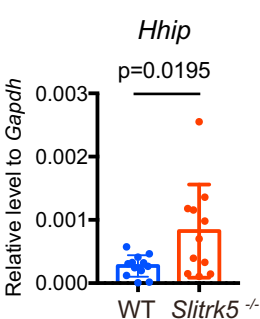
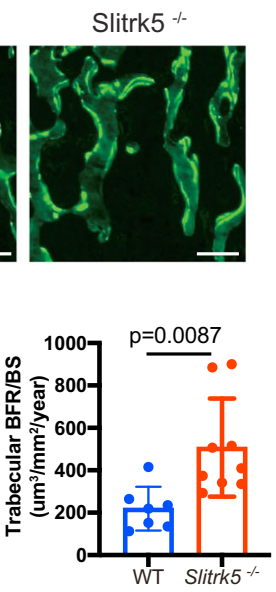

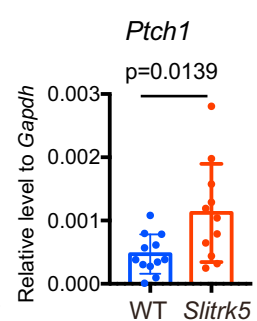

e

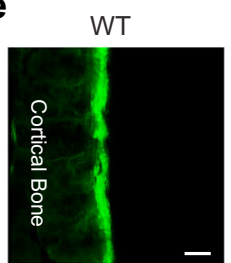

f

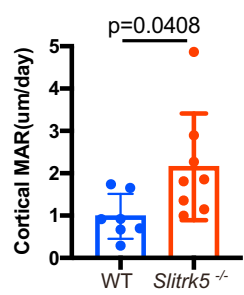

Slitrk5

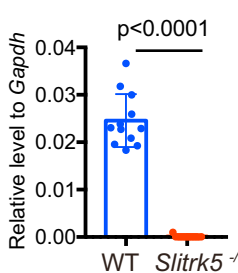

Slitrk5
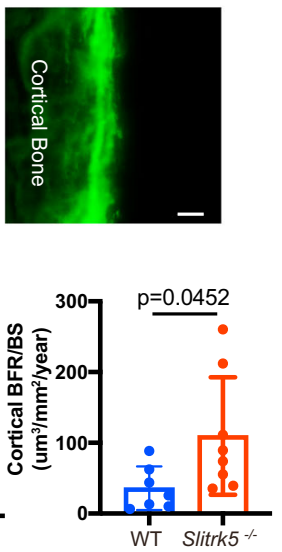

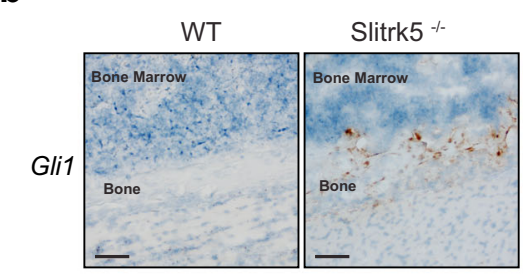

g

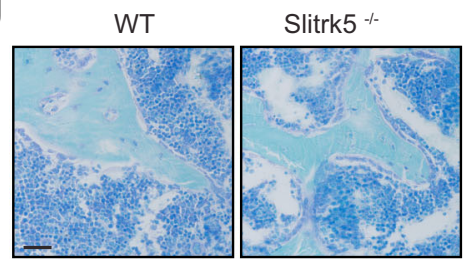

h

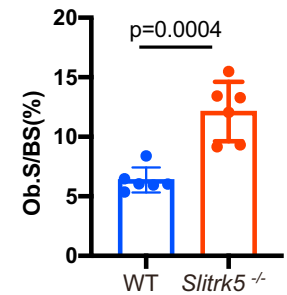

k
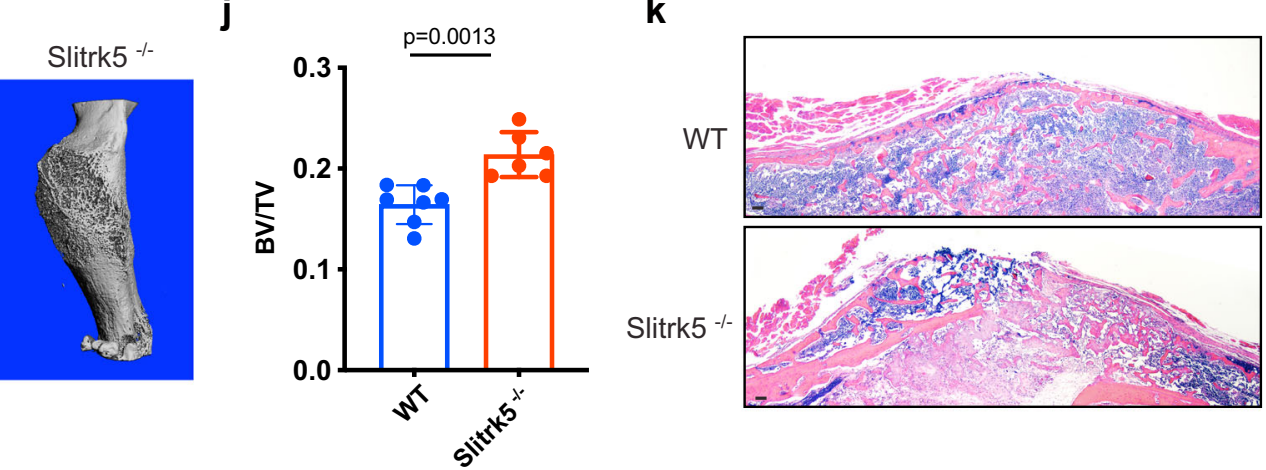

$$
\text { I }
$$

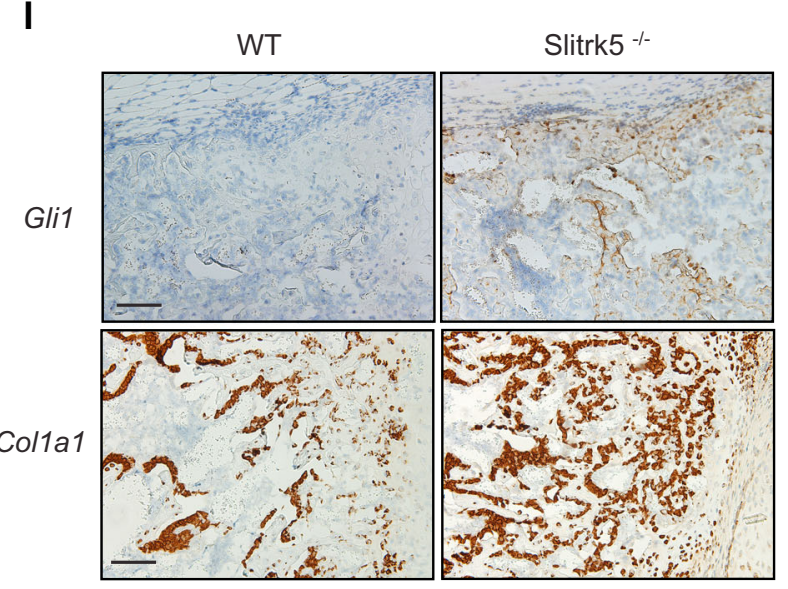

Slitrk5 $5^{-1-}$ mice as indicated by the upregulation of Gli1 expression (Fig. 5l, m). Taken together, loss of Slitrk5 led to increased bone formation in both physiological conditions and enhanced fracture healing, phenotypes consistent with biochemical observations that SLITRK5 is a negative regulatory coreceptor in the Hh pathway in osteoblasts. m
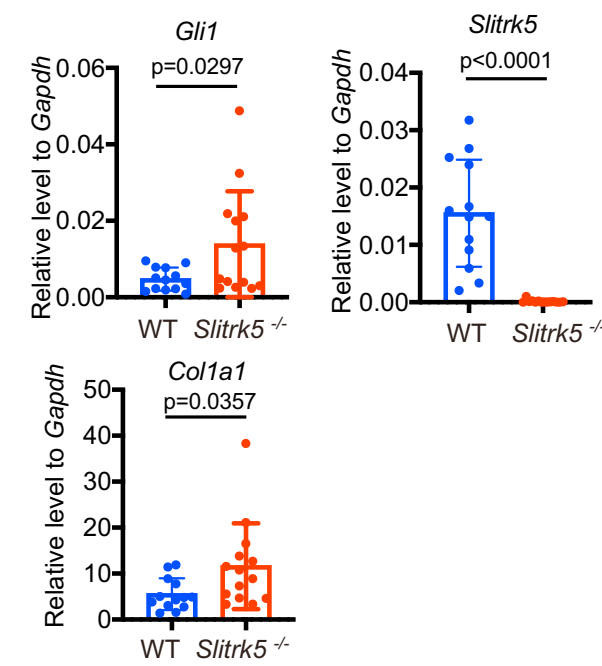

\section{Discussion}

Hh signaling is progressively decreased as osteoblasts mature. This prevents excessive bone resorption as activation of Hh signaling in mature osteoblasts upregulates RANKL expression which drives osteoclastogenesis and bone resorption ${ }^{7}$. Osteoblast responsiveness to Hh ligands is thus tightly controlled by multiple 
Fig. 5 Slitrk5 deficient mice display increased bone formation. a RT-PCR analysis of hedgehog signaling related gene expression in the tibias of 8-week-old WT and Slitrk5 ${ }^{-/} . n=11$ or 12 per group. Two-tailed unpaired $t$ test. b RNA in situ hybridization analysis using Gli7 probe in the tibias of WT and Slitrk5 $5^{-/-}$mice at 4 day old. Data are representative of two independent experiments, scale bar $=200 \mu \mathrm{m}$. c-f Calcein double labeling (c, e) and quantification of histomorphometric parameters $(\mathbf{d}, \mathbf{f})$ of the L3 vertebrae trabecular bone $(\mathbf{c}, \mathbf{d})$ and cortical bone $(\mathbf{e}, \mathbf{f})$ in 7 -week-old WT and Slitrk5-/- female mice. Mineral apposition rate (MAR, $\mu \mathrm{m} /$ day), bone formation rate/bone surface (BFR/BS) $\left(\mathrm{mm}^{3} / \mathrm{mm}^{2} /\right.$ year). N=7-9 per group. Data are presented as mean $\pm \mathrm{s}$.d, two-tailed unpaired $t$ test, data in (c, e) are representative of 7 (WT) or 9 (Slitrk5 $5^{-/-}$) independent samples. Scale bar $=250 \mu \mathrm{m}(\mathbf{c})$ and $25 \mu \mathrm{m}(\mathbf{e})$. $\mathbf{g}$, $\mathbf{h}$ Toluidine blue staining (g) and quantification of Ob.S/BS (h) of the L3 vertebrae in WT and Slitrk5 ${ }^{-/-}$female mice at 7 week old. Osteoblast surface/bone surface (Ob.S/BS), $n$ $=6$ per group. Data are presented as mean $\pm \mathrm{s} . \mathrm{d}$, two-tailed unpaired $t$ test, data in $(\mathbf{g})$ are representative of six independent samples, scale bar $=100 \mu \mathrm{m}$. $\mathbf{i}$ Representative $\mu \mathrm{CT}$ 3D images of mouse femurs at 21 days after open femoral midshaft fracture, scale bar $=1 \mathrm{~mm}$. $\mathbf{j} \mu \mathrm{CT}$ measurement of BV/TV in callus area in WT and Slitrk5 ${ }^{-/-}$mice at 21 days post-surgery. $N=6$ or 7 per group. Data are presented as mean \pm s.d, two-tailed unpaired $t$ test. $\mathbf{k}$ Representative H\&E staining images of fracture callus from in WT and Slitrk5 $5^{-/}$mice at 21 days after an open femoral midshaft fracture. Data are representative of three independent experiments, scale bar $=100 \mu \mathrm{m}$. I RNA in situ hybridization analysis using Gli1 and Colla1 probes in the callus area of WT and Slitrk5-/- mice at 12 days postsurgery. Data are representative of two independent experiments, scale bar $=100 \mu \mathrm{m}$. $\mathbf{m}$ RT-PCR analysis of Glit, Colla1, and Slitrk5 expression in the fracture callus from WT and Slitrk5 $5^{-/-}$mice at 12 days after open femoral midshaft fracture, $n=13$ or 14 per group. Two-tailed unpaired $t$ test.

levels of regulation. Gnas inhibits Hh signaling partially through activating the hedgehog inhibitor protein kinase A (PKA). Loss of Gnas increases Hh signaling, leading to enhanced osteoblast differentiation $^{21}$. Another regulator named speckle-type POZ protein $(S p o p)$ is an E3-ubiquitin ligase adapter, regulating the ubiquitination and degradation of GLI $3^{22}$. Spop-deficient osteoblasts showed increased GLI3 repressor levels and decreased Hh signaling, resulting in osteoblast differentiation defects ${ }^{23}$. Here, we identify Slitrk5 as a novel regulator of $\mathrm{Hh}$ signaling in osteoblasts. Slitrk5 acts at the most proximal steps in Hh signaling, acting upstream of SMO at the level of $\mathrm{SHH}$ and the $\mathrm{PTCH} 1$ receptor complex, enhancing $\mathrm{SHH}$-induced osteoblast differentiation.

Here we identify SLITRK5 as a Hh co-receptor that acts at the level of the primary $\mathrm{Hh}$ receptor $\mathrm{PTCH} 1$ to regulate $\mathrm{Hh}$ signaling. SLITRK5, therefore, joins other Hh co-receptors including BOC, $\mathrm{CDO}$, and GAS1 ${ }^{24-26}$. Hedgehog interacting protein (HHIP) attenuates $\mathrm{Hh}$ signaling by competing with $\mathrm{PTCH} 1$ for binding to $\mathrm{HH}$ ligands ${ }^{27-29}$. HHIP is induced by $\mathrm{Hh}$ signaling, forming a negative regulatory feedback loop ${ }^{27}$. Similarly, Glypican-3 (GPC3) competes with PTCH1 for Hh ligand binding, inhibiting $\mathrm{Hh}$ signaling ${ }^{30}$. The binding affinity of SLITRK5 for $\mathrm{SHH}$ $\left(K_{\mathrm{d}} \approx 40 \mathrm{nM}\right)$ is similar to that of GPC3 for SHH $\left(K_{\mathrm{d}} \approx 32 \mathrm{nM}\right)$, arguing that SLITRK5 binds to Hh ligands at a physiologically relevant concentration. Both of these negative regulatory coreceptors have slightly lower affinity than that of positive regulatory co-receptors such as HHIP-SHH $\left(K_{\mathrm{d}} \approx 6 \mathrm{nM}\right)^{29,30}$, which would suggest that with increasing ligand titration, first positive and then negative-regulatory pathways are engaged. This would allow productive signaling to be engaged first and then negative regulatory pathways are increasingly recruited with higher ligand concentrations. In addition, Slitrk5 is also transcriptionally upregulated by $\mathrm{Hh}$ signaling, suggesting that SLITRK5 forms a negative regulatory feedback loop in response to Hh stimulation. This also fits the overall pattern that many Hh pathway components, such as Gli1 and Ptch1, are themselves upregulated in response to $\mathrm{Hh}$ pathway activity.

$C d o$ and $B o c$ displayed different expression patterns during mouse embryonic development and mice with mutation of $C d o$ exhibited holoprosencephaly while Boc mutants showed defective commissural axon guidance $26,31-33$, suggesting the regulation of $\mathrm{Hh}$ signaling is cell context-dependent and $\mathrm{Hh}$ co-receptors may serve to provide tissue and context-specific "tuning" of $\mathrm{Hh}$ responsiveness. In line with this, we show here that, outside of the central nervous system (CNS), Slitrk5 expression is largely restricted to osteoblasts. Accordingly, Slitrk $5^{-1-}$ mice displayed increased bone formation but no other obvious $\mathrm{Hh}$ signaling related phenotypes outside of bone.
Given that Slitrk5 is also expressed in neural tissues, it is possible that Slitrk5 also modulates Hh signaling in neural cells and is therefore relevant to the oncogenesis of Hh pathway driven CNS tumors such as medulloblastoma. Supporting this, Slitrk5 expression is upregulated in tumors marked by active Hh signaling, such as $\mathrm{Hh}$ dependent medulloblastoma ${ }^{16}$. Similarly, missense mutations in SHH and Slitrk5 have been implicated in the attention deficit hyperactivity disorder ${ }^{34,35}$, raising the possibility that SLITRK5-regulation of Hh signaling contributes to disease processes outside of bone.

There are currently very limited therapeutic drug options for bone repair, thus it is notable both that prior studies identify $\mathrm{Hh}$ as a key pathway regulating fracture repair ${ }^{36,37}$ and that this study nominates SLITRK5 as a candidate therapeutic target relevant to this context. Hh signaling is activated during fracture healing and expression of a constitutively active SMO mutant in Coll-cre targeted osteoblasts led to enhanced bone formation in the fracture callus, suggesting that augmented Hh signaling can promote fracture healing ${ }^{19,36}$. A decrease in total $\mathrm{Hh}$ signaling activity has also been implicated as contributing to the age-related declines in fracture healing capacity and local osteoblast generation ${ }^{37}$. In addition, the hedgehog agonist purmorphamine enhanced bone regeneration in a calvarial defect mouse model ${ }^{20,36}$. Meanwhile, we here report that deletion of the negative Hh regulator Slitrk5 improved bone fracture healing. Thus, Hh signaling may have therapeutic importance for fracture healing. Given the narrow and osteoblast selective expression pattern of Slitrk5 alongside the absence of $\mathrm{Hh}$ associated phenotypes in nonskeletal tissues, targeting SLITRK5 is promising for offering an osteoblast-specific means to modulate $\mathrm{Hh}$ responses to enhance osteoblast differentiation and skeletal repair.

\section{Methods}

Mice. Slitrk $5^{-/-}$mice were previously described ${ }^{12}$. All experiments were performed according to the guidelines approved by the Animal Care and Use Committee of the Weill Cornell Medical College. All relevant ethical regulations have been complied with for animal testing and research. All mice were maintained in $12 / 12$ light/dark cycle at room temperature of $20.5-22.5^{\circ} \mathrm{C}$ and humidity of $30-70 \%$ and had ad libitum access to dry laboratory food and water.

ELISA assay. hSLTRIK5-ECD $(2 \mu \mathrm{g} / \mathrm{ml})$ or BSA $(2 \mu \mathrm{g} / \mathrm{ml})$ was added to the 96 well microtiter plates. The plates were incubated at room temperature for $2 \mathrm{~h}$ before being washed four times. SHH-His (1314-SH-025, R\&D systems) or control His peptide was added and the plates were incubated for another $2 \mathrm{~h}$ at room temperature. After 4 times wash, the plates were incubated at room temperature for $30 \mathrm{~min}$. After 4 times wash, substrate solution was added, and Relative Light Units was measured at $450 \mathrm{nM}$ using a luminometer.

Surface plasmon resonance. Surface plasmon resonance experiments were performed using a Sierra Sensors MASS-1 (Bruker Daltonics, Billerica, MA). 
Human SLITRK5 (2587-SK, R\&D Systems, Minneapolis, MN) was immobilized on a research-grade high-capacity amine sensor chip (Bruker Daltonics, Billerica, MA) using the amine coupling method. SHH was diluted in PBS-T ( $135 \mathrm{mM}$ sodium chloride, $2.7 \mathrm{mM}$ potassium chloride, $4.3 \mathrm{mM}$ Sodium Phosphate, $1.4 \mathrm{mM}$ potassium phosphate, $0.05 \%$ Tween- $20, \mathrm{pH} 7.4$ ) and flowed over the surface at a rate of $10 \mu \mathrm{l} / \mathrm{min}$.

Real time PCR analysis. For analysis of gene expression, total RNA from cultured cells or tissues was extracted using TRIzol(Qiagen) according to the manufacturer's instructions. cDNA was then obtained using a High-Capacity cDNA Reverse Transcription Kit (Invitrogen). An SYBR Green polymerase chain reaction (PCR) Master Mix Kit (Applied Biosystems) was used for real-time PCR. QuantStudio 6 Flex RT-PCR Software v1.3 was used for mRNA analysis. Sequences of the PCR primers used are shown in Supplementary Table 1.

Luciferase reporter assay. C3H10T1/2 cells were transiently transfected with GLI1 responsive reporter plasmid (GLI1-BS-Luc) and Renilla luciferase plasmid together with control or Slitrk5 plasmid. Twenty-four hour after transfection, cells were treated with vehicle or SHH in serum-free medium for $36 \mathrm{~h}$. Cells were then lysed and luciferase activity was measured using the Dual-luciferase reporter assay system (Promega)

Histomorphometry. Mice were injected with $20 \mathrm{mg} / \mathrm{kg}$ calcein (Sigma) at 10 days and 2 days before scarification. Plastic embedding, TRAP staining, and toluidine blue staining of the undecalcified lumbar region were performed as previously described ${ }^{38}$. Static and dynamic histomorphometric analyses were performed using the Osteomeasure Analysis System (Osteometrics).

RNA in situ hybridization. To detect Gli1 and Col1a1 RNA in formalin-fixed, paraffin-embedded tissues, ISH was performed using the BOND RNAscope Detection Reagents kit (DS9790, Leica Biosystems, Buffalo Grove, IL, USA) according to the manufacturer's instructions. Briefly, $20 \mathrm{ZZ}$ probe pairs targeting the Gli1 and Colla1 mRNA were designed and synthesized by Advanced Cell Diagnostics (catalog numbers 311008 and 537048). Tissue samples were incubated with Leica Epitope Retrieval 2 for $20 \mathrm{~min}$ at $95^{\circ} \mathrm{C}$, then pre-treated using Leica Protease at $40^{\circ} \mathrm{C}$ for $20 \mathrm{~min}$, then incubated with Glil or Collal RNA probe at $40^{\circ}$ C for 240 mins. ACD AMP $1-6$ was applied at $40^{\circ} \mathrm{C}$ for $60-120$ min for signal amplification before application of 3,3'-Diaminobenzidine (DAB).

Histology and immunostaining. Hind limbs from mice were dissected and fixed in ice-cold $4 \%$ paraformaldehyde solution for overnight and then decalcified in 0.5 M EDTA solution at $4{ }^{\circ} \mathrm{C}$ for 2 weeks. Samples were either embedded in optimal cutting temperature compound (Leica) and sectioned at $20 \mu \mathrm{m}$ thickness or embedded in paraffin and cut into 5 - $\mu \mathrm{m}$-thick sections. Immunostaining for $\beta$ galactosidase and OPN was performed using an anti- $\beta$-galactosidase antibody (GTX77365; GeneTex) and an anti-OPN antibody (AF808, R\&D Systems). For cilium staining, bone marrow stromal cells were harvested from 3-week-old mice and osteoblast differentiation was induced with ascorbic acid and $\beta$ -

glycerophosphate. Cells were then infected with lentiviral constructs encoding Slitrk5-flag, Ptch1-flag, or Smo-GFP. Cells were cultured under serum starvation conditions (medium with $0.5 \%$ fetal bovine serum (FBS)) for $48 \mathrm{~h}$ before $4 \mathrm{~h} \mathrm{SHH}$ $(300 \mathrm{ng} / \mathrm{ml})$ treatment. After SHH treatment, cells were fixed with $4 \%$ paraformaldehyde (PFA) for $10 \mathrm{~min}$, permeabilized with $0.1 \%$ (v/v) Triton X-100 for 10 min, blocked with $5 \%$ for $1 \mathrm{hr}$. Antibodies were used as follow: rabbit anti-Flag (1:500, cell signaling technology, 2368S) and mouse anti-acetylated tubulin (1:1000, Sigma, T7451). Imaging was performed with a Zeiss LSM 880 with an Airyscan high-resolution-detector confocal microscope. Carl Zeiss Zen 2.3 SP1 FP3 (black, v14.0.18.201, Germany) was used for immunofluorescence imaging analysis. To quantify the relative fluorescence of protein in the primary cilia, the CiliaQ Image plugins(CiliaQ-0.1.4,CiliaQ Editor_JNH-0.1.0 and CiliaQ Preparator_JNH-0.1.0) were used for image segmentation and ciliary fluorescence quantification ${ }^{39}$. For each cell, the ciliary fluorescence intensity was normalized to cell body intensity.

Osteoblasts culture and differentiation assays. Primary osteoblasts were isolated from 5-7 days old mice by triple collagenase/Dispase II digestion. Cells were cultured in $\alpha$-MEM medium (Gibco) containing 10\% FBS, 2 mM L-glutamine, $1 \%$ penicillin/streptomycin, $1 \%$ HEPES, and $1 \%$ nonessential amino acids, and differentiated with ascorbic acid and $\beta$-glycerophosphate. For ALP activity, osteoblast number was assessed quantitatively using Alamar blue assay. Cells were then washed, fixed in $10 \%$ neutral-buffered formalin, and incubated with a solution containing $6.5 \mathrm{mM} \mathrm{Na}_{2} \mathrm{CO}_{3}, 18.5 \mathrm{mM} \mathrm{NaHCO} 3,2 \mathrm{mM} \mathrm{MgCl}$, and phosphatase substrate (Sigma-Aldrich). ALP activity was measured by a luminometer. For extracellular matrix mineralization staining, cells were fixed in $10 \%$ neutralbuffered formalin and stained with alizarin red.

Immunoprecipitation and immunoblotting. HEK293T cells or C3H10T1/2 cells were transfected with the indicated DNA plasmids. Cells were lysed in RIPA buffer or TNT lysis buffer [ $10 \mathrm{mM}$ Tris, $50 \mathrm{mM} \mathrm{NaCl}, 5 \mathrm{mM}$ EDTA, $2 \mathrm{mM} \mathrm{NaF}, 30 \mathrm{mM}$ sodium pyrophosphate, $100 \mathrm{mM} \mathrm{Na}_{3} \mathrm{VO}_{4}, 0.5 \mathrm{mM}$ PMSF, $1 \mu \mathrm{g} / \mathrm{ml}$ leupeptin, $5 \mu \mathrm{g} / \mathrm{ml}$ aprotinin, and $1 \%$ Triton X100]. Flag or HA agaroses were used in the immunoprecipitation and immunoprecipitated proteins were then subjected to sodium dodecyl sulfate-polyacrylamide gel electrophoresis and transferred to Immobilon-P membranes (Millipore, Billerica, MA, USA). The membranes were then blocked with $5 \%$ nonfat milk and incubated with specific antibodies.

Antibodies and reagents. BDNF (248-BD-005/CF, R\&D systems) was used at a concentration of $40 \mathrm{ng} / \mathrm{ml}$. Purmorphamine (540220-5MG) was purchased from EMD Millipore K252a (11338) was purchased from Cayman. SHH (8908SH) was purchased from R\&D systems. EZview" Red Anti-HA Affinity Gel (E6779, Sigma), EZview $^{\text {"w }}$ Red ANTI-FLAG ${ }^{\oplus}$ M2 Affinity Gel (F2426, Sigma), Flag peptide (F3290, Sigma) and HA peptide (I2149, Sigma) were used for immunoprecipitation experiments. The following primary antibodies were used: anti-acetylated tubulin (Sigma, T7451, clone 6-11b-1,0000106162, 1:1000), anti-tubulin (Santa Cruz, sc23948,H160,C2316, 1:5000), anti-HA (cell signaling technology, 3724, C29F4, 12/ 2017, 1:2000), anti-DYKDDDDK (cell signaling technology, 14793s, D6W5B, 11/ 2018, 1:2000), anti-GFP (Abcam, ab13970, GR89472-25,1:5000), anti- $\beta$ galactosidase antibody (GTX77365; GeneTex,1:100), anti-SHH (H-160, sc-9024, Santa Cruz,1:1000), anti-SHH (E1, sc-365112, Santa Cruz,1:1000), antiOsteopontin (AF808-SP, R\&D systems1:400) and anti-Flag (9696S, Cell Signaling Technology, 1:1000). Cell lines were purchased from ATCC: C3H10T1/2 cells clone 8 (CCL-226), 293T (ATCC ${ }^{\oplus}$ CRL-3216 ${ }^{\mathrm{m}}$ ), Saos-2 (ATCC ${ }^{\oplus}$ HTB-85").

Bone fracture model. Surgery was performed under isoflurane $(2.5 \%)$ anesthesia via nosecone. The surgical sites were sterilized, and an incision was made on the right femur. The fracture was made using a Dremel saw with a diamond thin cutting wheel (Cat. \#100230-724; VWR) and a 25-gauge syringe needle was inserted from femoral condyles to stabilize the femur. Muscles were reapproximated, and the skin was closed using wound clips.

MicroCT analysis. The mouse femurs were skinned and fixed in $70 \%$ ethanol Femurs were scanned using a Scanco Medical uCT 35 system with a spatial resolution of $7 \mu \mathrm{m}$. The X-ray tube potential of $55 \mathrm{kVp}$, an xray intensity of 0.145 $\mathrm{mA}$, and an integration time of $600 \mathrm{~ms}$. For analysis of femoral bone mass, a region of trabecular bone $2.1 \mathrm{~mm}$ wide was contoured, starting 280 microns from the proximal end of the distal femoral growth plate. For analysis of fracture callus bone volume, the whole callus was contoured.

Statistics. All results are presented as the mean \pm SD. Comparisons between two groups were analyzed using a two-tailed, unpaired Student's $t$ test. One-way Anova followed by Tukey's post hoc test was used when the data involves multiple group comparisons. GraphPad PRISM v.8.4.3 was used for statistical analysis.

Reporting summary. Further information on research design is available in the Nature Research Reporting Summary linked to this article.

\section{Data availability}

All relevant data are available from the authors. Uncropped blots are provided in Supplementary Fig. 5. Source data are provided with this paper.

Received: 9 July 2020; Accepted: 9 July 2021;

Published online: 29 July 2021

\section{References}

1. Ganesan K., Jandu J. S., Roane D. Osteoporosis (Secondary). In StatPearls (2020)

2. Pak, E. \& Segal, R. A. Hedgehog signal transduction: key players, oncogenic drivers, and cancer therapy. Dev. Cell 38, 333-344 (2016).

3. Briscoe, J. \& Therond, P. P. The mechanisms of Hedgehog signalling and its roles in development and disease. Nat. Rev. Mol. Cell Biol. 14, 416-429 (2013).

4. St-Jacques, B., Hammerschmidt, M. \& McMahon, A. P. Indian hedgehog signaling regulates proliferation and differentiation of chondrocytes and is essential for bone formation. Genes Dev. 13, 2072-2086 (1999).

5. Ohba, S. et al. Patched1 haploinsufficiency increases adult bone mass and modulates Gli3 repressor activity. Dev. Cell 14, 689-699 (2008).

6. Kitaura, Y. et al. Gli1 haploinsufficiency leads to decreased bone mass with an uncoupling of bone metabolism in adult mice. PLoS ONE 9, e109597 (2014)

7. Mak, K. K. et al. Hedgehog signaling in mature osteoblasts regulates bone formation and resorption by controlling PTHrP and RANKL expression. Dev. Cell 14, 674-688 (2008). 
8. Aruga, J. \& Mikoshiba, K. Identification and characterization of Slitrk, a novel neuronal transmembrane protein family controlling neurite outgrowth. Mol. Cell Neurosci. 24, 117-129 (2003).

9. Linhoff, M. W. et al. An unbiased expression screen for synaptogenic proteins identifies the LRRTM protein family as synaptic organizers. Neuron 61, 734-749 (2009).

10. Abelson, J. F. et al. Sequence variants in SLITRK1 are associated with Tourette's syndrome. Science 310, 317-320 (2005).

11. Katayama, K. et al. Disorganized innervation and neuronal loss in the inner ear of Slitrk6-deficient mice. PLoS ONE 4, e7786 (2009).

12. Shmelkov, S. V. et al. Slitrk5 deficiency impairs corticostriatal circuitry and leads to obsessive-compulsive-like behaviors in mice. Nat. Med. 16, 598-602 (2010). 591p following 602 .

13. $\mathrm{Xu}, \mathrm{R}$. et al. Targeting skeletal endothelium to ameliorate bone loss. Nat. Med. 24, 823-833 (2018).

14. Wu, C. et al. BioGPS: an extensible and customizable portal for querying and organizing gene annotation resources. Genome Biol. 10, R130 (2009).

15. Song, M. et al. Slitrk5 mediates BDNF-dependent TrkB receptor trafficking and signaling. Dev. Cell 33, 690-702 (2015).

16. Mao, J. et al. A novel somatic mouse model to survey tumorigenic potential applied to the Hedgehog pathway. Cancer Res. 66, 10171-10178 (2006).

17. Spinella-Jaegle, S. et al. Sonic hedgehog increases the commitment of pluripotent mesenchymal cells into the osteoblastic lineage and abolishes adipocytic differentiation. J. Cell Sci. 114, 2085-2094 (2001).

18. Alman, B. A. The role of hedgehog signalling in skeletal health and disease Nat. Rev. Rheumatol. 11, 552-560 (2015).

19. Horikiri, Y. et al. Sonic hedgehog regulates osteoblast function by focal adhesion kinase signaling in the process of fracture healing. PLOS ONE $\mathbf{8}$, e76785 (2013).

20. Lee, C.-S. et al Smoothened agonist sterosome immobilized hybrid scaffold for bone regeneration. Sci. Adv. 6, eaaz7822 (2020).

21. Regard, J. B. et al. Activation of Hedgehog signaling by loss of GNAS causes heterotopic ossification. Nat. Med 19, 1505-1512 (2013).

22. Wang, C., Pan, Y. \& Wang, B. Suppressor of fused and Spop regulate the stability, processing and function of Gli2 and Gli3 full-length activators but not their repressors. Development 137, 2001-2009 (2010).

23. Cai, H. \& Liu, A. Spop promotes skeletal development and homeostasis by positively regulating Ihh signaling. Proc. Natl Acad. Sci. USA 113 , 14751-14756 (2016).

24. Izzi, L. et al. Boc and Gas1 each form distinct Shh receptor complexes with Ptch1 and are required for Shh-mediated cell proliferation. Dev. Cell 20, 788-801 (2011).

25. Allen, B. L. et al. Overlapping roles and collective requirement for the coreceptors GAS1, CDO, and BOC in SHH pathway function. Dev. Cell 20 775-787 (2011).

26. Okada, A. et al. Boc is a receptor for sonic hedgehog in the guidance of commissural axons. Nature 444, 369-373 (2006).

27. Chuang, P. T. \& McMahon, A. P. Vertebrate Hedgehog signalling modulated by induction of a Hedgehog-binding protein. Nature 397, 617-621 (1999).

28. Bosanac, I. et al. The structure of SHH in complex with HHIP reveals a recognition role for the Shh pseudo active site in signaling. Nat. Struct. Mol. Biol. 16, 691-697 (2009).

29. Bishop, B. et al. Structural insights into hedgehog ligand sequestration by the human Hedgehog-interacting protein HHIP. Nat. Struct. Mol. Biol. 16 698-703 (2009).

30. Capurro, M. I. et al. Glypican-3 inhibits Hedgehog signaling during development by competing with patched for Hedgehog binding. Dev. Cell 14, 700-711 (2008).

31. Seppala, M. et al. Gas1 is a modifier for holoprosencephaly and genetically interacts with Sonic Hedgehog. J. Clin. Investig. 117, 1575-1584 (2007).

32. Zhang, W., Kang, J. S., Cole, F., Yi, M. J. \& Krauss, R. S. Cdo functions at multiple points in the Sonic Hedgehog pathway, and Cdo-deficient mice accurately model human holoprosencephaly. Dev. Cell 10, 657-665 (2006).

33. Tenzen, T. et al. The cell surface membrane proteins Cdo and Boc are components and targets of the Hedgehog signaling pathway and feedback network in mice. Dev. Cell 10, 647-656 (2006).

34. Heussler, H. S., Suri, M., Young, I. D. \& Muenke, M. Extreme variability of expression of a Sonic Hedgehog mutation: attention difficulties and holoprosencephaly. Arch. Dis. Child 86, 293-296 (2002).
35. Salesse, C. et al. Opposite control of excitatory and inhibitory synapse formation by slitrk 2 and slitrk 5 on dopamine neurons modulates hyperactivity behavior. Cell Rep. 30, 2374-2386.e2375 (2020).

36. Baht, G. S., Silkstone, D., Nadesan, P., Whetstone, H. \& Alman, B. A. Activation of hedgehog signaling during fracture repair enhances osteoblasticdependent matrix formation. J. Orthop. Res. 32, 581-586 (2014).

37. Matsumoto, K. et al. Expression and role of sonic hedgehog in the process of fracture healing with aging. Vivo 30, 99-105 (2016).

38. Fukuda, T. et al. Sema $3 \mathrm{~A}$ regulates bone-mass accrual through sensory innervations. Nature 497, 490-493 (2013).

39. Hansen J. N., Rassmann S., Stüven B., Jurisch-Yaksi N. \& Wachten D. CiliaQ-a simple, open-source software for automated quantification of ciliary morphology and fluorescence in 2D, 3D, and 4D images. Eur. Phys. J. E 44, 18 (2021).

\section{Acknowledgements}

This project was supported by a Career Award for Medical Scientists from the Burroughs Wellcome Fund and the NIH under awards DP5OD021351 and R01AR075585. This publication is based on research supported by the Pershing Square Sohn Cancer Research Alliance via an award to M.B.G. R.X. is supported by the National Key R\&D Program of China (2020YFA0112900) and the National Natural Science Foundation of China (81972034 and 92068104 to R.X.). We thank Pathology Core Facilities at Weill Cornell Medicine for their technical assistance.

\section{Author contributions}

Conception and design: M.B.G., R.X., Y.S. and J.S.; development of methodology and acquisition of data: R.X., J.S., D.Y.S., M.E., A.R.Y., N.L., S.L., S.B., S.D., T.E.W., A.G.K. and I.C.L.; analysis and interpretation of data: M.B.G., R.X., D.Y.S. and J.S.; writing and revision of the paper: M.B.G., R.X., J.S., A.R.Y., Z.L., M.C., S.J.M., F.S.L. and J.H.S.; study supervision: M.B.G.; All authors reviewed and gave final approval to the paper.

\section{Competing interests}

J.H.S. is a scientific co-founder of AAVAA therapeutics and holds equity in this company. The remaining authors declare no competing interests.

\section{Additional information}

Supplementary information The online version contains supplementary material available at https://doi.org/10.1038/s41467-021-24819-w.

Correspondence and requests for materials should be addressed to R.X. or M.B.G.

Peer review information Nature Communications thanks Benjamin Allen and the other anonymous, reviewer(s) for their contribution to the peer review of this work. Peer reviewer reports are available.

Reprints and permission information is available at http://www.nature.com/reprints

Publisher's note Springer Nature remains neutral with regard to jurisdictional claims in published maps and institutional affiliations.

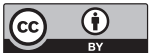

Open Access This article is licensed under a Creative Commons Attribution 4.0 International License, which permits use, sharing, adaptation, distribution and reproduction in any medium or format, as long as you give appropriate credit to the original author(s) and the source, provide a link to the Creative Commons license, and indicate if changes were made. The images or other third party material in this article are included in the article's Creative Commons license, unless indicated otherwise in a credit line to the material. If material is not included in the article's Creative Commons license and your intended use is not permitted by statutory regulation or exceeds the permitted use, you will need to obtain permission directly from the copyright holder. To view a copy of this license, visit http://creativecommons.org/ licenses/by/4.0/.

(C) The Author(s) 2021 